\title{
Critical behavior and net-charge fluctuations from lattice QCD*
}

\section{Frithjof Karsch}

Fakultät für Physik, Universität Bielefeld, D-33615 Bielefeld, Germany

Physics Department, Brookhaven National Laboratory, Upton, NY 11973, USA

E-mail: karschephysik.uni-bielefeld.de

\begin{abstract}
We present recent results on the critical and pseudo-critical temperatures in $(2+1)$-flavor QCD with a physical strange quark mass and two degenerate light quark masses extrapolated to the chiral limit and tuned to the physical value, respectively. We furthermore discuss implication of the observed low chiral phase transition temperature, $T_{c}^{0}=132_{-6}^{+3} \mathrm{MeV}$, for the structure of cumulants of conserved charge fluctuations at vanishing baryon chemical potential and consequences for the possible location of the QCD critical endpoint in the QCD phase diagram at non-zero baryon chemical potential.
\end{abstract}

Corfu Summer Institute 2018 "School and Workshops on Elementary Particle Physics and Gravity" (CORFU2018)

31 August - 28 September, 2018

Corfu, Greece

*Based on talks given at the conference "The Critical Point and Onset of Deconfinement" (CPOD 2018), Sept. 24-28, 2018, Corfu, Greece; the EMMI workshop "Probing the Phase Structure of Strongly Interacting Matter: Theory and Experiment", March 25-29, 2019, GSI Darmstadt, Germany, and the EMMI Rapid Task Force meeting "Dynamics of critical fluctuations: theory-phenomenology-HIC", April 8-12, 2019, GSI Darmstadt, Germany. 


\section{Introduction}

Understanding the phase structure of strongly interacting matter is one of the central goals in studies of the properties of strong interaction matter at finite temperature and density through largescale numerical calculations in the framework of lattice regularized Quantum Chromo Dynamics (QCD). Also experimentally major efforts at the Large Hadron Collider (LHC) at CERN and the Relativistic Heavy Ion Collider (RHIC) at Brookhaven National Laboratory are devoted to this goal.

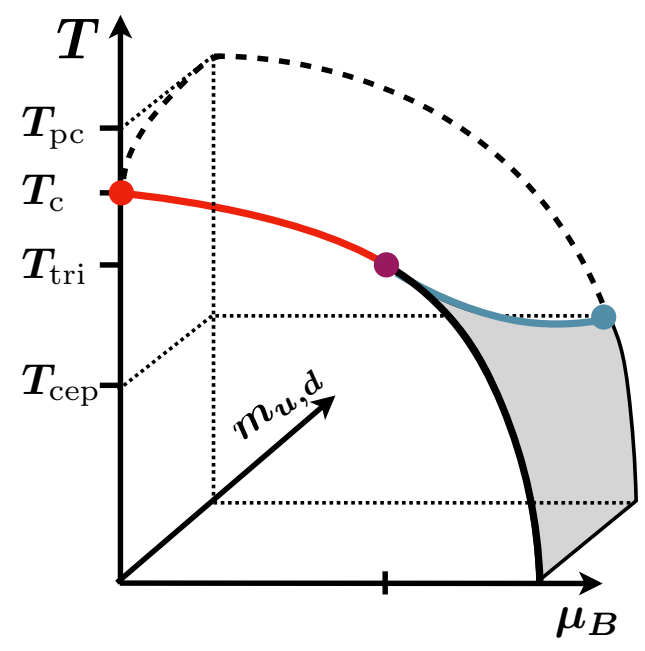

Figure 1: Sketch of a possible QCD phase diagram in the space of temperature $(T)$, baryon chemical potential $\left(\mu_{B}\right)$ and light quark masses $\left(m_{u, d}\right)$.

At vanishing net baryon-number density or, equivalently, vanishing baryon chemical potential $\left(\mu_{B}\right)$, it is by now well established that the transition from hadronic matter at low temperature to the quark-gluon plasma at high temperature is a continuous (crossover) transition taking place at a pseudo-critical temperature $T_{p c}$ (for recent reviews see [1, 2]). While this is the case for physical values of the quark masses, it is expected that in the limit of vanishing light quark masses $\left(m_{u, d}\right)$ strong interaction matter shows true critical behavior resulting from the appearance of second order phase transitions at some temperature $T_{c}\left(\mu_{B}\right)$. In QCD with two massless quark flavors this transition is due to the spontaneous breaking of the $S U_{L}(2) \times$ $S U_{R}(2) \simeq O(4)$ chiral symmetry [3] and persists as such also at non-zero baryon chemical potential.

At non-zero values of the two light quark masses the transition is only a smooth crossover for small values of $\mu_{B}$. At larger $\mu_{B}$, however, it is expected that a second order phase transition arises at the endpoint $\left(T_{c e p}\right)$ of a line of first order transitions, at which the net baryon-number density changes discontinuously [4]. Critical behavior in the vicinity of this endpoint will be controlled by the 3-d, $Z(2)$ universality class. This Ising-like transition will exist for arbitrary values of the light quark masses and thus will meet the $O(4)$ chiral transition line at $m_{u, d}=0$ in a tri-critical point $\left(T_{t r i}\right)$. A sketch of the resulting phase diagram, which also indicates the relative ordering of the various transition temperatures, is shown in Fig. 1. This generic phase diagram, in particular the indicated ordering of the various characteristic (phase) transition temperatures, is in qualitative agreement with various model calculations $[4,5,6]$.

In the following we will present recent lattice QCD results on the pseudo-critical $\left(T_{p c}\right)$ and critical temperature $\left(T_{c}\right)$ in $(2+1)$-flavor QCD at $\mu_{B}=0$. We relate these findings to the structure of higher order cumulants of conserved charge fluctuations, and discuss how they constrain the location of a possible critical point at $\mu_{B}>0$ and physical values of the quark masses. 

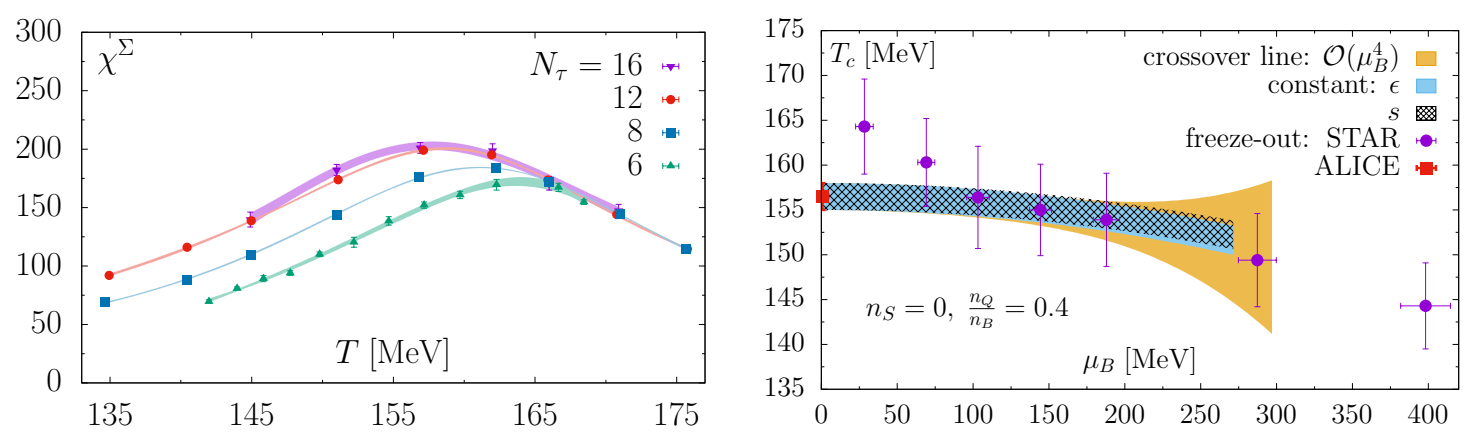

Figure 2: Left: The chiral susceptibility $\left(\chi^{\Sigma} \equiv \chi_{M}\right)$ calculated on lattices with different temporal extent $N_{\tau}$ for physical values of the degenerate light $(u, d)$ and strange quark masses. Right: Crossover temperature $T_{p c}\left(\mu_{B}\right)$ determined from continuum extrapolated results for the location of peaks in the chiral susceptibilities defined in Eq. 2.2 and some further observables introduced in Ref. [7]. Also shown in this figure are lines of constant energy and entropy density [8] as well as results for freeze-out temperatures determined from data on particle yields measured by the STAR and ALICE collaborations $[9,10]$.

\section{Universal pseudo-critical and critical behavior}

\subsection{Pseudo-critical temperature in (2+1)-flavor QCD}

In the limit of vanishing up and down quark masses QCD possesses an exact global symmetry, the chiral $S U_{L}(2) \times S U_{R}(2)$ flavor symmetry. This symmetry is spontaneously broken at low temperature, signaled by a non-vanishing chiral condensate $(\langle\bar{\psi} \psi\rangle)$. Chiral symmetry is explicitly broken due to the non-vanishing light quark masses. Nonetheless, this explicit breaking is small enough for chiral symmetry providing a good, approximate order parameter at non-zero temperature - the chiral condensate $\langle\bar{\psi} \psi\rangle$. Its variation with quark mass as well as temperature is large in a small temperature interval, which leads to well defined peaks in the corresponding chiral $\left(\chi^{\Sigma}\right)$ and mixed $\left(\chi_{t}\right)$ susceptibilities. These maxima in the susceptibilities are used to define pseudo-critical temperatures, which, in the limit of vanishing quark masses, converge to the uniquely defined critical temperature for the chiral phase transition.

For our studies of the chiral phase transition we use as an order parameter for chiral symmetry breaking

$$
\Sigma=\frac{1}{f_{K}^{4}}\left[m_{s}\left(\langle\bar{\psi} \psi\rangle_{u}+\langle\bar{\psi} \psi\rangle_{d}\right)-\left(m_{u}+m_{d}\right)\langle\bar{\psi} \psi\rangle_{s}\right],
$$

where $\langle\bar{\psi} \psi\rangle_{f}=T\left(\partial \ln Z / \partial m_{f}\right) / V$ denotes chiral condensates of the up $(u)$, down $(d)$, and strange $(s)$ quarks. A fraction of the strange quark chiral condensates is subtracted from the light quark chiral condensates in order to eliminate ultra-violet divergences, linear in the quark masses, and the condensates are multiplied with the strange quark mass in order to define a renormalization group invariant observable. The kaon decay constant $f_{K}$ is used to set the scale and define a dimensionless order parameter $\Sigma$ (sometimes also denoted as $M$ ).

Pseudo-critical temperatures are extracted from the location of peaks in the chiral and mixed 

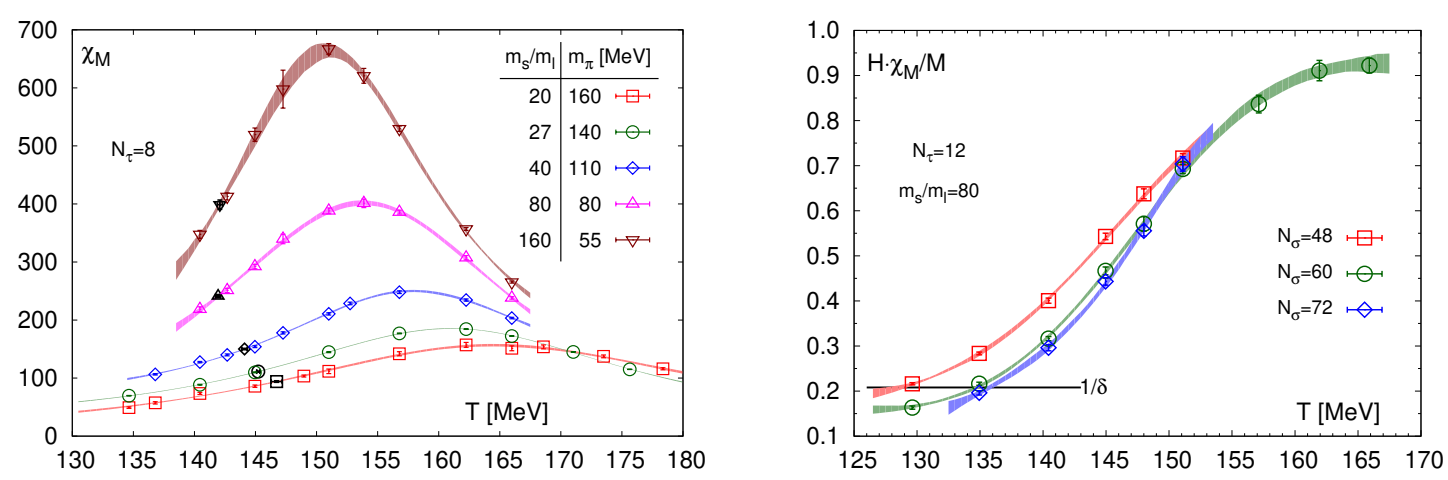

Figure 3: Left: The chiral susceptibility for several values of the quark mass ratio $H=m_{l} / m_{s}$ on lattices with temporal extent $N_{\tau}=8$ and spatial lattice sizes that are varied in the range $N_{\sigma}=(4-7)$ when going from the largest to the smallest light quark mass value. Right: The ratio $H \chi_{M} / M$ for $H=1 / 80$ and $N_{\tau}=12$ for three different spatial lattice sizes $N_{\sigma}$.

susceptibilities

$$
\begin{aligned}
\chi_{M} & =m_{s}\left(\frac{\partial}{\partial m_{u}}+\frac{\partial}{\partial m_{d}}\right) \Sigma, \\
\chi_{t} & =T \frac{\mathrm{d}}{\mathrm{d} T} \Sigma .
\end{aligned}
$$

For different values of the lattice spacing, $a=1 / T N_{\tau}$, the peak locations in different susceptibilities are determined. From an extrapolation to the continuum limit, that takes into account $\mathscr{O}\left(a^{2}\right)$ cutoff effects one then determines pseudo-critical temperatures for the chiral transition. Results from a recent determination of pseudo-critical temperatures at physical values of the light and strange quark masses are shown in Fig. 2. The left hand figure shows the chiral susceptibility $\left(\chi^{\Sigma} \equiv\right.$ $\left.\chi_{M}\right)$ calculated on different size lattices $\left(N_{\sigma}^{3} N_{\tau}\right.$, with $\left.N_{\sigma}=4 N_{\tau}\right)$ [7] using the Highly Improved Staggered Quark (HISQ) action [11]. Other observables, e.g. the mixed susceptibility $\chi_{t}$, yield pseudo-critical temperatures, which in the continuum limit differ from each other by less than $2 \mathrm{MeV}$ [7]. For the pseudo-critical temperature this analysis yields,

$$
T_{p c}=(156.5 \pm 1.5) \mathrm{MeV} .
$$

A comparison of this pseudo-critical temperature with the freeze-out temperature determined from data on particle yields in heavy ion collisions at the LHC [9] suggests that the formation of hadrons after the cooling of the expanding hot and dense quark-gluon matter created in these collisions does take place close to the phase boundary characterized by this pseudo-critical temperature (see Fig. 2 (right)).

\subsection{Critical temperature in (2+1)-flavor QCD}

An analogous analysis can be performed for other values of the light quark masses $\left(m_{l} \equiv\right.$ $\left.\left(m_{u}+m_{d}\right) / 2\right)$, keeping the strange quark mass fixed at its physical value. The approach to the chiral limit, $H \equiv m_{l} / m_{s} \rightarrow 0$, can then be examined by monitoring the quark mass dependence of the chiral order parameter and its susceptibility $\left(\chi_{M}\right)$. Some results for the quark mass dependence 
of $\chi_{M}$, calculated with the HISQ action, are shown in Fig. 3 (left) [12]. For sufficiently small values of the light quark masses and close to the chiral transition temperature, i.e. in the scaling regime, the peak location in $\chi_{M}$, and similarly in $\chi_{t}$, is controlled by universal scaling functions,

$$
\chi_{M}(T, H) \sim h^{1 / \delta-1} f_{\chi}(z)+\text { regular }, \chi_{t}(T, H) \sim h^{1 / \delta-1 / \beta \delta} f_{G}^{\prime}(z)+\text { regular },
$$

where $\beta$ and $\delta$ are critical exponents for the universality class of the chiral transition, $z \equiv z_{0}[(T-$ $\left.\left.T_{c}^{0}\right) / T_{c}^{0}\right] / H^{1 / \beta \delta}, h=H / h_{0}$ and $h_{0}, z_{0}$ are non-universal constants. The peak locations in $\chi_{M}$ and $\chi_{t}$ are related to maxima of the scaling functions $f_{\chi}(z)$ and $f_{G}^{\prime}(z)$, respectively. The quark mass dependence of pseudo-critical temperatures thus is controlled by the scaling variable $z$. The increase of the peaks is controlled by the prefactors. As can be seen in Fig. 3 (left) the peak in $\chi_{M}$ increases rapidly with decreasing quark, or equivalently pion, mass and the peak location shifts towards smaller values of the temperature. In the scaling regime, close to the chiral limit, contributions from regular terms will be small and one expects to find

$$
T_{p c}(H)=T_{c}^{0}\left(1+\frac{z_{X}}{z_{0}} H^{1 / \beta \delta}\right)
$$

with $z_{X}$ being a universal constant defining the location of the maximum in $\chi_{X}$, e.g. $X \equiv M$ or $t$ when using the peak locations of $\chi_{M}$ and $\chi_{t}$ defined in Eq. 2.2 and Eq. 2.3, respectively. For the 3- $d, O(4)$ universality class one has, $z_{M} \simeq 1.4(1), z_{t} \simeq 0.8(2)$, and $1 / \beta \delta \simeq 0.55$ [13]. As $z_{0}$ typically is of $\mathscr{O}(1)$, Eq. 2.6 suggests that the pseudo-critical temperatures determined from the peak locations in $\chi_{M}$ and $\chi_{t}$ will show a rather strong dependence on the light quark masses. In fact, QCD-inspired model calculations $[14,15]$ suggest that $T_{c}^{0}$ might be $(20-30) \mathrm{MeV}$ smaller than $T_{p c}$ calculated for physical values of the quark masses, for which $H \simeq 1 / 27$.

In order to determine the chiral phase transition temperature $T_{c}^{0}$ it thus would be advantageous to use observables which similarly to the maxima in susceptibilities correspond to a fixed value of the scaling variable $z$, but are related to a value $z \equiv z_{X}$ that is close to zero. Two such observables have been utilized recently [12] for this purpose. One may define two characteristic temperatures, $T_{\delta}$ and $T_{60}$, through the relations

$$
\begin{aligned}
\frac{H \chi_{M}\left(T_{\delta}\right)}{M\left(T_{\delta}\right)} & =\frac{1}{\delta} \\
\chi_{M}\left(T_{60}\right) & =0.6 \chi_{M}^{\text {peak }} .
\end{aligned}
$$

In the thermodynamic limit the corresponding scaling variables $z_{\delta}$ and $z_{60}$ both are close to zero. The resulting estimators, $T_{\delta}$ and $T_{60}$, for the chiral phase transition temperature are quark mass dependent only due to the presence of contributions arising from regular terms in the partition function. They therefore provide good estimators for the chiral phase transition temperature. Some results for the ratio $H \chi_{M} / M$, from which the estimator $T_{\delta}$ is extracted, are shown in Fig. 3 (right). When decreasing the quark masses towards the chiral limit finite volume effects increase and some care needs to be taken in the extrapolation to the thermodynamic limit. After (i) infinite volume, (ii) continuum, and (iii) chiral limit extrapolations these estimators yield for the chiral phase transition temperature [12]

$$
T_{c}^{0}=132_{-6}^{+3} \mathrm{MeV}
$$


The chiral phase transition temperature thus is about $25 \mathrm{MeV}$ smaller than the pseudo-critical temperature extracted from the location of the peak in the chiral susceptibility. As will be discussed further in Section 3, this has consequences also for the phase transition temperature $T_{c e p}$ at which a possible critical point at physical values of the light quark masses and at non-zero values of the baryon chemical potential may occur.

\subsection{Curvature of the phase transition line in the chiral limit}

Close to the chiral limit, in the vicinity of the critical temperature, the non-analytic (singular) behavior of the logarithm of the partition function, i.e. the pressure, is described by a scaling function, $f_{s}(z)$. Deviations from scaling are given in terms of an analytic (regular) function $f_{r}$,

$$
\frac{P}{T^{4}}=h^{2-\alpha} f_{s}(z)+f_{r}\left(T, \mu_{B}, \mu_{Q}, \mu_{S}, m_{f}\right),
$$

The reduced temperature variable $t$ entering the scaling variable $z \sim t / h^{1 / \beta \delta}$ will also dependent on the chemical potentials. In leading order, and for vanishing strangeness and electric charge chemical potentials, one has

$$
t \sim \frac{T-T_{c}^{0}}{T_{c}^{0}}+\kappa_{2}^{B, 0}\left(\frac{\mu_{B}}{T}\right)^{2},
$$

which also reflects the temperature dependence of the chiral phase transition temperature, $T_{c}\left(\mu_{B}\right)=$ $T_{c}^{0}\left(1-\kappa_{2}^{B, 0}\left(\mu_{B} / T\right)^{2}\right)$.

At physical values of the quark masses the curvature of the transition line, $\kappa_{2}^{B}$, will in general differ from $\kappa_{2}^{B, 0}$, receiving corrections from regular terms, terms arising from universal correctionsto-scaling or higher order terms in the scaling variables being proportional to $H\left(T-T_{c}^{0}\right)$. This curvature term can be extracted from the $\mu_{B}$-dependence of the location of maxima of various susceptibilities. Using a Taylor expansion of, e.g. the mixed chiral susceptibility $\chi_{t}\left(T, \mu_{B}\right)$ in terms of temperature and baryon chemical potential around the pseudo-critical point $\left(T_{p c}, \mu_{B}=0\right)$, one obtains for the curvature $\kappa_{2}^{B}[7]$,

$$
\kappa_{2}^{B}=\left.\frac{1}{2 T^{2} \partial_{T}^{2} \chi_{t}}\left[T \partial_{T} \chi_{t}^{\prime}-2 \chi_{t}^{\prime}\right]\right|_{\left(T_{p c}, \mu_{B}=0\right)},
$$

with $\chi_{t}^{\prime}=T^{2} \partial^{2} \chi_{t} / \partial \mu_{B}^{2}$. Similarly one can derive expressions for higher order expansion coefficients of $T_{p c}\left(\mu_{B}\right)$. The analysis performed in Ref. [7] gave $\kappa_{2}^{B}=0.015(4)$ in agreement with other recent determinations of the leading order correction to $T_{p c}[16,17]$. The next-to-leading order correction, $\kappa_{4}^{B}$, is an order of magnitude smaller and consistent with zero within current statistical errors. The resulting $\mu_{B}$-dependence of the crossover line for physical quark masses is shown in Fig. 2 (right).

In the limit of vanishing quark mass the curvature coefficients $\kappa_{2}^{B}$ will approach the corresponding curvature term of the chiral phase transition line, $\kappa_{2}^{B, 0}$. In fact, in the absence of contributions from regular terms the curvature coefficient will be quark mass independent, as seen from the general scaling ansatz given in Eq. 2.10. To what extent this holds true may be probed by comparing temperature and chemical potential derivatives of $P / T^{4}$. In the absence of substantial 
contributions from regular terms one expects to find in the scaling regime,

$$
\frac{T^{2}}{2} \frac{\partial^{2} \Sigma}{\partial \mu_{B}^{2}}=\kappa_{2}^{B, 0} T_{c}^{0} \frac{\partial \Sigma}{\partial T}
$$

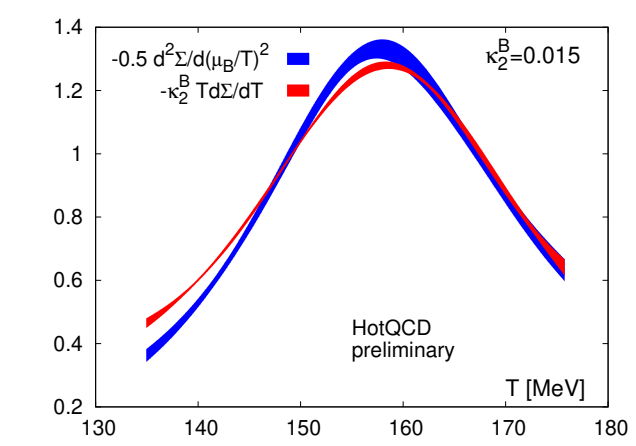

Figure 4: Derivatives of the chiral order parameter with respect to temperature and baryon chemical potentials, respectively. Shown are results for $N_{\tau}=12$.

A test of this relation is shown in Fig. 4, where $\kappa_{2}^{B, 0} \equiv \kappa_{2}^{B}$ has been assumed. This indeed suggests that the curvature of the chiral phase transition line is similar in magnitude to that of the pseudo-critical line at physical values of the quark masses.

\section{Higher order cumulants in the crossover region}

The sketch of the QCD phase diagram shown in Fig. 1, which qualitatively is consistent with model calculations for the quark mass dependence of transition lines in the QCD phase diagram $[4,5,6]$, suggests that a possible critical point at physical values of the quark masses is located at a temperature $T_{c e p}$ below the chiral phase transition temperature $T_{c}^{0}$. If this is correct, it has significant consequences also for the properties of higher order cumulants of conserved charge fluctuations.

Cumulants of conserved charge fluctuations, evaluated at vanishing chemical potentials $\left(\mu_{B, Q, S}\right)$, appear as expansion coefficients in Taylor series for thermodynamic quantities. The relative magnitude of subsequent expansion coefficients controls the convergence of these expansions and determines their radius of convergence. The pattern of sign changes in these expansion coefficients provides information on the location of singularities in the plane of complex-valued chemical potentials which cause the breakdown of the Taylor expansions. E.g., for a series of the form $\sum_{x} c_{n} x^{n}$ the singularity determining the radius of convergence lies on the real-x axis, if an $n_{0}$ exists such that all expansion coefficients $c_{n}$ are positive for all $n>n_{0}$ [18] (see also discussion in [19]). Only in this case the radius of convergence can be unambiguously related to the existence of a phase transition in the thermodynamic system under consideration. One thus may examine the sign of subsequent expansion coefficients and their relative magnitude in order to judge whether or not the convergence of a Taylor series is limited by the appearance of a phase transition for some real-valued chemical potential.

At small values of the chemical potentials the QCD partition function may be expanded in a Taylor series. E.g. the pressure can be written as

$$
\frac{P}{T^{4}}=\frac{1}{V T^{3}} \ln Z\left(T, V, \hat{\mu}_{u}, \hat{\mu}_{d}, \hat{\mu}_{s}\right)=\sum_{i, j, k=0}^{\infty} \frac{\chi_{i j k}^{B Q S}}{i ! j ! k !} \hat{\mu}_{B}^{i} \hat{\mu}_{Q}^{j} \hat{\mu}_{S}^{k},
$$


with $\chi_{000}^{B Q S} \equiv P(T, 0) / T^{4}$ and $\hat{\mu}_{X}=\mu_{X} / T$. The generalized susceptibilities are given as derivatives of $P / T^{4}$ at vanishing values of the conserved charge chemical potentials,

$$
\chi_{i j k}^{B Q S} \equiv \chi_{i j k}^{B Q S}(T)=\left.\frac{\partial P(T, \hat{\mu}) / T^{4}}{\partial \hat{\mu}_{B}^{i} \partial \hat{\mu}_{Q}^{j} \partial \hat{\mu}_{S}^{k}}\right|_{\hat{\mu}=0} .
$$

If, at some value of the temperature, the radius of convergence of the Taylor series for the pressure arises from a singularity in the complex- $\mu$ plane, one should find that Taylor expansion coefficients will have an irregular sign structure, i.e. at this temperature positive and negative expansion coefficients will appear in the Taylor series. Such changes of sign are indeed observed for various cumulants of conserved charge fluctuations, starting with sixth order expansion coefficients. Although not rigorous in the mathematical sense stated above, these sign changes suggest that Taylor expansions in this temperature range are not limited by a physical singularity related to a phase transition, but by some singularity in the complex- $\mu$ plane.

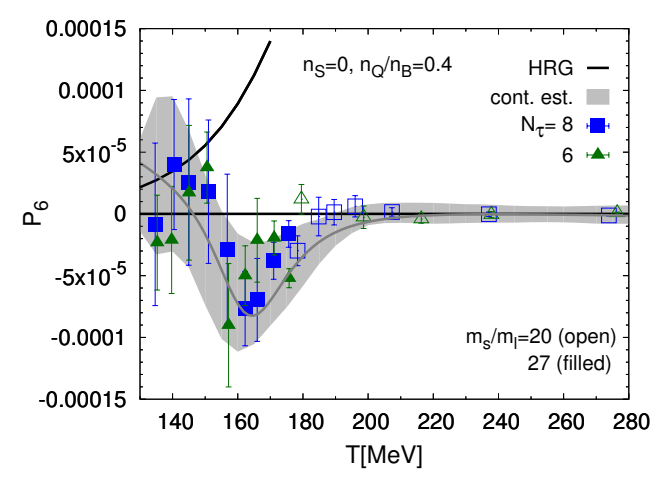

Figure 5: Temperature dependence of the sixth order expansion coefficient of the pressure in $(2+1)$-flavor QCD at vanishing net strangeness and fixed electric charge to baryon number density, $n_{Q} / n_{B}=0.4$ [8].

In Fig. 5 we show the sixth order expansion coefficient of the pressure for the case of vanishing net strangeness and a fixed electric charge to baryon-number density, $n_{Q} / n_{B}=$ 0.4 [8],

$$
\begin{aligned}
\frac{P}{T^{4}}= & P_{0}+P_{2} \hat{\mu}_{B}^{2}+P_{4} \hat{\mu}_{B}^{4}+P_{6} \hat{\mu}_{B}^{6} \\
& +\mathscr{O}\left(\hat{\mu}_{B}^{8}\right) .
\end{aligned}
$$

While the expansion coefficients up to $\mathscr{O}\left(\mu_{B}^{4}\right)$ are all positive [8], the sixth order expansion coefficient, $P_{6}$, starts to change sign with increasing temperature, i.e. $P_{6}<0$ for $T \gtrsim 150 \mathrm{MeV}$. These sign changes are expected to become more frequent and start at lower temperatures in higher orders of the expansion.

The irregular sign structure becomes more apparent in simpler cumulants like the net up-quarknumber cumulants, which are statistically easier to control. Up to eight order cumulants are shown in Fig. 6 (left). As can be seen, the sign of $\chi_{n+2}^{u}(T)$ can be deduced from the temperature derivative of $\chi_{n}^{u}(T)$, as suggested by Eq. 2.11. Similar behavior is found for the expansion coefficients of the quadratic net electric charge fluctuations at non-zero baryon chemical potential,

$$
\chi_{2}^{Q}\left(T, \mu_{B}\right)=\chi_{02}^{B Q}(T)+\frac{1}{2} \chi_{22}^{B Q}(T) \hat{\mu}_{B}^{2}+\frac{1}{24} \chi_{42}^{B Q}(T) \hat{\mu}_{B}^{4}+\mathscr{O}\left(\mu_{B}^{6}\right),
$$

where, for simplicity, we have set $\mu_{Q}=\mu_{S}=0$. The first three expansion coefficients are shown in Fig. 6 (right). We note that $\chi_{42}^{B Q}$ vanishes at the temperature where $\chi_{22}^{B Q}$ has its maximum. Also these expansion coefficients thus seem to be in accordance with the pattern resulting from Eq. 2.11 in the scaling regime, i.e. two derivatives with respect to the baryon chemical potential are proportional to a single derivative with respect to temperature. This leads to the expectation 

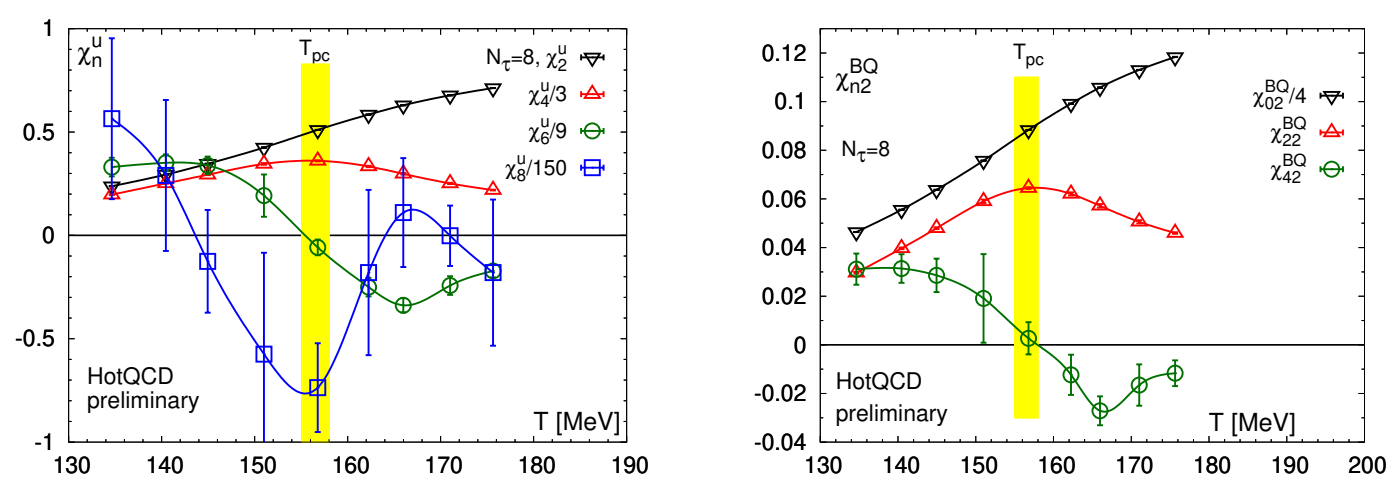

Figure 6: Left: Temperature dependence of up to eight order cumulants of net up-quark-number fluctuations calculated on lattices with temporal extent $N_{\tau}=8$ Right: Expansion coefficients of net electric charge fluctuations for the case of vanishing electric charge and strangeness chemical potentials. In both figures the lines are smooth spline interpolations drawn to guide the eye.

that the eight order cumulants, $\chi_{62}^{B Q}$, will be negative in the temperature range $T \in[135 \mathrm{MeV}$ : $165 \mathrm{MeV}$. At high temperature subsequent expansion coefficients thus show an irregular sign structure, which is in accordance with the expectation that for physical quark mass values a possible critical endpoint in the QCD phase diagram will be located at a temperature below the chiral phase transition temperature $T_{c}^{0}$.

\section{Conclusions}

New results on the chiral phase transition temperature $T_{c}^{0}$ in $(2+1)$-flavor QCD suggests that this temperature is well below the pseudo-critical temperature $T_{p c}$ at physical values of the light and strange quark masses. Moreover, it is found that many $6^{\text {th }}$ and higher order cumulants of conserved charge fluctuations are no longer strictly positive but start showing an irregular sign structure at temperatures $T \gtrsim T_{c}^{0}$. This suggests that a possible second order phase transition at physical values of the quark masses and for non-vanishing baryon chemical potential can occur only at a temperature $T_{c e p}<T_{c}^{0}$, if it exists at all.

\section{Acknowledgement}

This work was supported in part through Contract No. DE-SC001270 with the U.S. Department of Energy, the Deutsche Forschungsgemeinschaft (DFG) through the CRC-TR 211 "Stronginteraction matter under extreme conditions", grant number 315477589 - TRR 211, and the grant 05P18PBCA1 of the German Bundesministerium für Bildung und Forschung.

\section{References}

[1] H.-T. Ding, F. Karsch and S. Mukherjee, Thermodynamics of strong-interaction matter from Lattice QCD, Int. J. Mod. Phys. E24 (2015) 1530007 [1504.05274].

[2] M. D'Elia, High-Temperature QCD: theory overview, Nucl. Phys. A982 (2019) 99 [1809.10660].

[3] R. D. Pisarski and F. Wilczek, Remarks on the Chiral Phase Transition in Chromodynamics, Phys. Rev. D29 (1984) 338. 
[4] A. M. Halasz, A. D. Jackson, R. E. Shrock, M. A. Stephanov and J. J. M. Verbaarschot, On the phase diagram of QCD, Phys. Rev. D58 (1998) 096007 [hep-ph/ 9804290 ].

[5] M. A. Stephanov, QCD critical point and complex chemical potential singularities, Phys. Rev. D73 (2006) 094508 [hep-lat/ 0603014$].$

[6] M. Buballa and S. Carignano, Inhomogeneous chiral phases away from the chiral limit, Phys. Lett. B791 (2019) 361 [1809.10066].

[7] A. Bazavov et al., Chiral crossover in QCD at zero and non-zero chemical potentials, 1812.08235.

[8] A. Bazavov et al., The QCD Equation of State to $\mathscr{O}\left(\mu_{B}^{6}\right)$ from Lattice $Q C D$, Phys. Rev. D95 (2017) $054504[1701.04325]$.

[9] A. Andronic, P. Braun-Munzinger, K. Redlich and J. Stachel, Decoding the phase structure of QCD via particle production at high energy, Nature 561 (2018) 321 [1710.09425].

[10] STAR collaboration, L. Adamczyk et al., Bulk Properties of the Medium Produced in Relativistic Heavy-Ion Collisions from the Beam Energy Scan Program, Phys. Rev. C96 (2017) 044904 [1701.07065].

[11] HPQCD, UKQCD collaboration, E. Follana, Q. Mason, C. Davies, K. Hornbostel, G. P. Lepage, J. Shigemitsu et al., Highly improved staggered quarks on the lattice, with applications to charm physics, Phys. Rev. D75 (2007) 054502 [hep-lat/ 0610092 ].

[12] H. T. Ding et al., The chiral phase transition temperature in (2+1)-flavor QCD, 1903.04801.

[13] J. Engels and F. Karsch, The scaling functions of the free energy density and its derivatives for the $3 d$ O(4) model, Phys. Rev. D85 (2012) 094506 [1105. 0584 ].

[14] J. Berges, D. U. Jungnickel and C. Wetterich, Two flavor chiral phase transition from nonperturbative flow equations, Phys. Rev. D59 (1999) 034010 [hep-ph/9705474].

[15] J. Braun, B. Klein, H. J. Pirner and A. H. Rezaeian, Volume and quark mass dependence of the chiral phase transition, Phys. Rev. D73 (2006) 074010 [hep-ph/ 0512274 ].

[16] R. Bellwied, S. Borsanyi, Z. Fodor, J. Günther, S. D. Katz, C. Ratti et al., The QCD phase diagram from analytic continuation, Phys. Lett. B751 (2015) 559 [1507. 07510].

[17] C. Bonati, M. D’Elia, F. Negro, F. Sanfilippo and K. Zambello, Curvature of the pseudocritical line in QCD: Taylor expansion matches analytic continuation, Phys. Rev. D98 (2018) 054510 [1805.02960].

[18] S. Gaunt and A. Gutmann, Phase Transitions and Critical Phenomena 3 (Edts. C. Domb and M.S. Green), Academic Press (1974) .

[19] C. R. Allton, M. Doring, S. Ejiri, S. J. Hands, O. Kaczmarek, F. Karsch et al., Thermodynamics of two flavor QCD to sixth order in quark chemical potential, Phys. Rev. D71 (2005) 054508 [hep-lat/0501030]. 